\title{
SonicHoop: Using Interactive Sonification to Support Aerial Hoop Practices
}

\author{
Wanyu Liu \\ abbywanyuliu@ircam.fr \\ ${ }^{1}$ STMS Lab, IRCAM, CNRS, \\ Sorbonne Université \\ Paris, France \\ ${ }^{2}$ Université Paris-Saclay, CNRS, Inria \\ Orsay, France
}

\author{
Artem Dementyev \\ artem.dementyev@gmail.com \\ Google Research \\ Mountain view, California, USA
}

\author{
Diemo Schwarz \\ Emmanuel Fléty \\ Diemo.Schwarz@ircam.fr \\ Emmanuel.Flety@ircam.fr \\ STMS Lab, IRCAM, CNRS, \\ Sorbonne Université \\ Paris, France
}

\author{
Wendy E. Mackay \\ Michel Beaudouin-Lafon \\ Université Paris-Saclay, CNRS, Inria \\ Orsay, France \\ mackay@lri.fr,mbl@lri.fr
}

Frédéric Bevilacqua

Frederic.Bevilacqua@ircam.fr

STMS Lab, IRCAM, CNRS,

Sorbonne Université

Paris, France
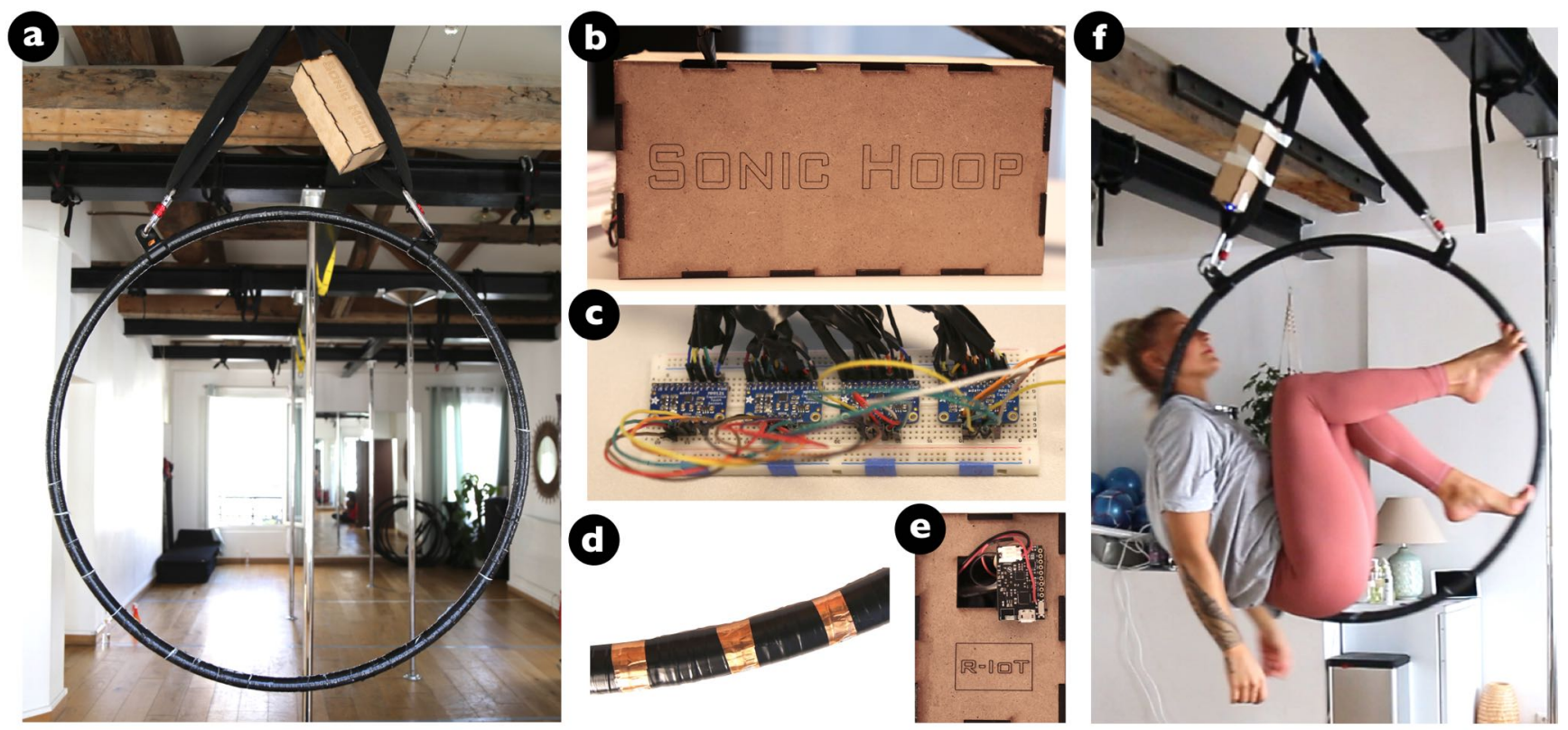

Figure 1: (a) SonicHoop suspended from the ceiling; (b) a laser cut wooden box containing (c) electronics, here a breadboard with 4 Arduino MPR121 capactive touch sensors; (d) electrodes on the hoop; (e) a Bitalino R-IoT wireless sensor sends touch event data to a laptop via wifi; (f) Participant 1 using SonicHoop $\odot$ Selma Marin.

\begin{abstract}
Aerial hoops are circular, hanging devices for both acrobatic exercise and artistic performance that let us explore the role of interactive sonification in physical activity. We present SonIcHoop,

CHI '21, May 8-13, 2021, Yokohama, Japan

(c) 2021 Association for Computing Machinery.

This is the author's version of the work. It is posted here for your personal use. Not for redistribution. The definitive Version of Record was published in CHI Conference on Human Factors in Computing Systems (CHI '21), May 8-13, 2021, Yokohama, Fapan, https://doi.org/10.1145/3411764.3445539.
\end{abstract}

an augmented aerial hoop that generates auditory feedback via capacitive touch sensing, thus becoming a digital musical instrument that performers can play with their bodies. We compare three sonification strategies through a structured observation study with two professional aerial hoop performers. Results show that SonIcHoop fundamentally changes their perception and choreographic processes: instead of translating music into movement, they search for bodily expressions that compose music. Different sound designs affect their movement differently, and auditory feedback, regardless 
of type of sound, improves movement quality. We discuss opportunities for using SoNicHoop as an aerial hoop training tool, as a digital musical instrument, and as a creative object; as well as using interactive sonification in other acrobatic practices to explore full-body vertical interaction.

\section{CCS CONCEPTS}

- Human-centered computing $\rightarrow$ Interaction devices; Soundbased input / output; Auditory feedback.

\section{KEYWORDS}

Interactive sonification; auditory feedback; sound; capacitive sensing; aerial hoop

\section{ACM Reference Format:}

Wanyu Liu, Artem Dementyev, Diemo Schwarz, Emmanuel Fléty, Wendy E. Mackay, Michel Beaudouin-Lafon, and Frédéric Bevilacqua. 2021. SonicHoop: Using Interactive Sonification to Support Aerial Hoop Practices. In CHI Conference on Human Factors in Computing Systems (CHI '21), May 8-13, 2021, Yokohama, Japan. ACM, New York, NY, USA, 16 pages. https: //doi.org/10.1145/3411764.3445539

\section{INTRODUCTION}

Rooted in the circus and the world of acrobatics, aerial hoop is a form of exercise that has only recently become accessible to the general public. An aerial hoop is a large steel ring suspended from the ceiling (Fig. 1a), in which an aerialist can practice spins, drops, tricks and positions. Aerial hoop demands intense physical exercise, requiring as much strength and full-body coordination as swimming [3] and yoga [84]. It is also an art form that requires flexibility, balance, elegance and concentration in order to learn long combinations of figures.

Similar to other movement-based activities, aerial hoop practices rely heavily on proprioception $[29,58]$. The aerialist perceives and acts on diverse sensorial information, including the body parts that are currently in contact with the instrument, the rotation speed of the aerial hoop given the aerialist's current posture and preceding figures, available as intrinsic feedback [47, 49], as well as their own movement quality and balance. To facilitate movement skill acquisition and playful experiences, the fields of Human-Computer Interaction (HCI), sports science and psychology have also proposed an increasing number of interactive design solutions that provide extrinsic or augmented feedback. This includes visual augmentation, e.g. [84], auditory augmentation, e.g. [2], haptic augmentation e.g. [82], and combinations of some [23], or all of them [3]. These interactive systems span many physical activities including yoga [84], rock climbing [38], snowboarding [82], skiing [30], swimming [3], squats and deadlifts [23], slackline [2], golf training [36], and dancing [66].

We are motivated to explore how augmented feedback, particularly interactive sonification, affects aerial hoop practices. First, augmented feedback, regardless of modality, can provide information about the aerialist's movements that is not otherwise available. We want to see how such information is used by aerialists and how it changes their practices. Second, it is practically impossible to use visual feedback, since the hoop rotates on itself all the time, making it difficult for aerialists to visually verify their movements using screens or projections. Similarly, it is difficult to provide haptic feedback as different body parts might be in contact with different parts of the hoop depending upon the figures (Figure $2 \mathrm{~b}$ ), some of which include large surfaces. Haptic feedback may therefore not be felt by the aerialist, or may incur discomfort. Third, using auditory feedback, in particular interactive sonification, has been relatively well established in sports science to increase performance, self-awareness and enjoyment [72]. Last but not least, we want to explore the possibility of transforming the aerial hoop into a digital musical instrument that can be played by the performer's body. Instead of the usual approach, where aerial hoop performances are choreographed to a piece of music, we are interested in reversing the process, where aerialists compose music through their movements on the hoop. This follows in the tradition of movement-based interactive sound environments that become digital music instruments or novel kinds of performing arts [35, 50]; and raises new challenges for the study of systems at the intersection of HCI, sports and music.

This paper introduces SoNicHoop, an augmented aerial hoop with capacitive touch sensing and interactive sonification. After reviewing related work, we outline our design considerations, and describe SonicHoop's hardware, software and sonification strategies. We present the results of a technical evaluation and a structured observation with two professional aerial hoop performers, and discuss the effects of different sounds on participants' movements and choreographic process. We conclude with future directions for using SoNICHoop in aerial hoop pedagogy and performance, as well as the potential of interactive sonification in other acrobatic practices.

\section{RELATED WORK}

Since the practice of aerial hoop is both a physical exercise and an art form, we review HCI studies and systems that support physical training, the use of interactive sonification in sports science, digital music and performing arts, as well as interactive sensing techniques applied to large surfaces.

\subsection{Systems to Support Physical Training in HCI}

The design of interactive systems in HCI addresses multiple facets of physical activity, from supporting correct movement execution, e.g. $[3,82]$, to enhancing playful experiences and bodily interaction, e.g. [51, 52], by augmenting users' visual, auditory and tactile perceptions. Visual augmentations are the most common: Kajastila et al. [38] project interactive graphics on an indoor climbing wall to motivate and instruct climbers; Vidal et al. introduce wearable projecting lights for yoga [84] and strength training exercises [85] to augment instructor's and trainee's movements; and Park et al. propose an interactive snowboard [61] and later a skateboard [62] with color-coded visual displays of the performer's weight distribution. Others have explored tactile and auditory augmentation: Spelmezan [82] investigates the use of tactile instructions for amateur snowboarders; Bial et al. [7] embed actuators in cyclists' shoes to communicate the rhythm of pedaling; Hasegawa et al. [30] sonify the center of gravity of skiers to help them control positions; and Nylander et al. [56] describe a system 
for experiencing a golf swing by generating an audio mirror of the movement of the golf club. Multimodal augmentation has also been studied in the context of swimming [3], skateboarding [63], and squats and deadlifts exercises [23].

Each modality has benefits and limitations given the context of use. For example, a positive aspect of visual augmentation is the ability to make complex movement features easier to understand, such as movement trajectory [63], weight distribution [61] and posture [84]. On the other hand, it risks distracting the user due to the dominance of the human visual system [88], and may lead users to depend on visual feedback at the expense of proprioception [73]. By contrast, the effects of auditory and tactile augmentation are peripheral and less obtrusive [57], but raise design challenges such as how to convey information effectively (see [81] for a review). SoNIcHoop uses interactive sonification to communicate and augment the aerialist's position on the aerial hoop.

\subsection{Interactive Sonification in Sports}

Researchers in sports science have long investigated interactive sonification to increase athletes' performance, self-awareness and engagement [72]. Their work has been motivated by neurobiological and behavioral studies that demonstrate the close interaction between auditory and motor areas of the brain, as well as the importance of auditory information in perceptual-motor processes. This work has addressed a wide range of physical activities, including swimming [14, 70,74], slackline [2], gymnastics performed on a pommel horse [4], running [8,9], speed skating [10, 11], aerobics [31], handball [32], squat jumping [55], precision shooting [41], trampoline [65], basketball [67], cycling [46, 80], muscular training (biceps curl) [89], weightlifting [45], golf [36], german wheel [34], and rowing $[21,71]$.

Many of these studies report improved performance in movement execution, control and learning [21, 74], which, while important goals for sports, are mostly irrelevant for musical aesthetics. These studies also differ in what they sonify and how, e.g. the actual movement [70] vs. errors or deviation from desired performance [10]. Most use a single sonification mode that maps one aspect of movement to one sound feature. After reviewing 179 publications related to sonification of physical quantities, Dubus and Bresin concluded that simple sound synthesis prevails: many use pure tones that vary in pitch and amplitude, often driven by position or velocity of the corresponding movements [20]. Few studies address how different sonification designs affect perception and emotion $[42,83]$. We adopt a direct sonification strategy that sonifies the performer's physical contact with the aerial hoop, and explore three different sound designs to determine how they affect the aerialist's practice.

\subsection{Interactive Sound Systems in Digital Music and Performing Arts}

The use of interactive sound systems and/or interactive sonification techniques is widely explored in digital music and performing arts where the goal is to produce new, unconventional or specific sounds. In addition to many commercially available touch surfaces, such as Sensel Morph [79], Linnstrument [43] and Continuum [15], musicians have also crafted a number of novel digital musical instruments that let them control sounds using bodily expressions. Many examples can be seen in the Guthman musical instrument competition [54], such as Laser Harp ${ }^{1}$. Art pieces that create sound using only the performer's body can also be seen in, e.g. Very Nervous System (1982-1991) by David Rokeby ${ }^{2}$ and Corpus Nil by Marco Donnarumma ${ }^{3}$. Sound and music communities such as SMC (Sound \& Music Computing) and NIME (New Interfaces for Musical Expression) also investigate research questions such as how to map movement parameters to sound synthesis parameters [35], how to control sound with gesture $[5,6]$, how to process audio interactively [75], and how to integrate machine learning into music making [24].

In addition to providing auditory feedback on aerial hoop movements, we also want to explore the possibility of transforming the aerial hoop into a digital musical instrument that can be played by the performer's body. In the artistic discipline of aerial hoop, the aerialist dances figures within the physical constraints of the hoop, which offers an interesting and novel area for further exploring interactive sonification.

\subsection{Interactive Sensing Techniques}

Finally, we review research projects that developed large touch and interactive sensing surfaces. We are inspired by previous work in capacitive sensing, as it is a promising technique for making large interactive objects. Researchers have demonstrated large and reconfigurable capacitive touch sensors, using roll-to-roll printing [26, 27]. Such sensors can be deployed quickly, are flexible, and can conform to the object's shape. Furthermore, the capacitive sensing matrix has been integrated into fabrics [64] or on-skin electronics [86]. Other contact sensing techniques have also shown promise for interactive surfaces. For example, Z-Tiles [68] uses Force Sensitive Resistors (FSR) to create interactive, pressure-sensitive floor tiles; SensorTape [17] uses an array of infrared proximity sensors to develop a quickly deployable interactive surface.

Other research has explored non-contact interactive sensing, such as using large electric field electrodes to detect nearby human movements [90]. The electric field approach has been successfully deployed in dance and sonification contexts [60]. A common noncontact approach is to use visual tracking with cameras, e.g. a depth camera such as Microsoft Kinect [37], infrared-based motion capture (e.g. OptiTrack System [59]), or Inertial Measurement Unit (IMU) suit [69]. We chose to use capacitive touch sensing to build the interactive aerial hoop based on our design process and choices, described in the next section.

\section{DESIGN CONSIDERATIONS}

This section describes the aerial hoop practices that inspired and challenged our design choices. This discipline sits between sports and arts, and involves an essential instrument - the hoop - which can be augmented. It provides a rich test bed for studying the role of interactive sonification in physical activity, here both athletic and artistic.

\footnotetext{
${ }^{1}$ https://www.youtube.com/watch?v=Rt99-MAu2GY

${ }^{2}$ https://vimeo.com/8120954

${ }^{3}$ https://vimeo.com/152710490
} 


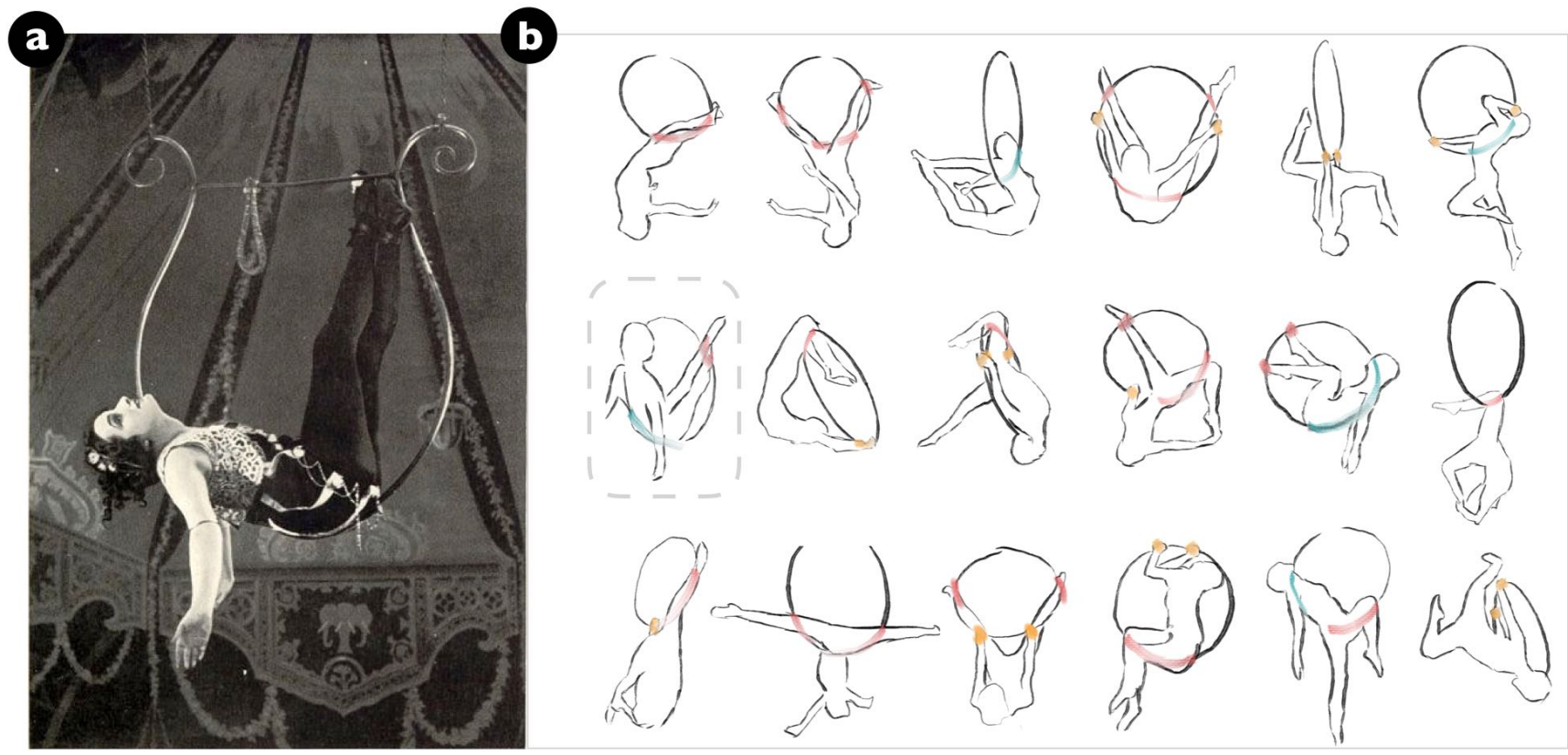

Figure 2: (a) Circus performer Caedo posing in a "Babe in the Moon" pose in this lyre-shaped apparatus in 1893; (b) Sketches of common aerial hoop figures. Contact between the aerial hoop and the performer's hands is highlighted in yellow; legs in red and upper body in green.

\subsection{Aerial Hoop Practices}

An aerial hoop, also known as a lyra, an aerial ring or a cerceau, is a circular steel hoop that is suspended from a height, using specialized rigging straps and a combination of carabiners and swivels. The word lyra comes from the Latin word for "lyre", which is a harp-like musical instrument that has a circular shape. The first recorded use of an aerial hoop was by a performer called "Caedo" in 1893 (Figure 2a). Cirque du Soleil [19] introduced the nowfamiliar circular form, and popularized it as an aerial art form in its shows in the mid-20th century. Now aerial hoop has transcended its stereotypical role as part of a circus act into special fitness programs offered in dance studios and gyms worldwide.

The practice of aerial hoop requires a combination of physical strength (pull-ups), flexibility, including leg flexibility (front and side splits) and back flexibility (similar to contortion), balance, memorization of a sequence of figures, and fluidity. The training often takes years. A typical aerial hoop class starts with warm-up exercises on the floor and on the hoop, and practices of a sequence of figures ${ }^{4}$. A sequence of figures on the hoop (Figure $2 b$ ) often starts from the floor, requiring pulling oneself up to sit on the hoop. One can go higher up with legs hanging on the upper part of the hoop and the rest of the body upside down. The aerial hoop also spins continuously, at a speed determined by the performer's current position and previous figures. To perform an aerial hoop piece, the aerialist often picks an existing piece of music that varies in tempo and energy so that the choreographed sequence of figures matches the characteristics of the music.

\footnotetext{
${ }^{4}$ An aerial figure is a specific position on the hoop.
}

\subsection{Design Goals}

We used a participatory design approach [53] that included aerial hoop practitioners throughout the design process. First, we conducted semi-structured interviews in a dance studio with aerial hoop students and teachers to understand their needs and expectations with respect to an interactive hoop. Second, we discussed with Optitrack [59] at SIGGRAPH 2019 [1] since they organized a live performance of aerial hoop ${ }^{5}$. We derived the following design goals from the analysis of interview results and discussions with Optitrack staff. Our four design goals are:

(1) to detect movements on the hoop so that various aerial figures are taken into account;

(2) to avoid forcing aerialists to wear heavy equipment;

(3) to preserve the hoop's existing physical properties; and

(4) to ensure the hoop is durable and affordable (if it becomes a product).

Among those interviewed, two professional aerial hoop performers/teachers were involved in the 6-month design process, regularly iterating with us on the design ideas, as well as on the engineering requirements, described in the next subsection. They also served as subject matter experts in the structured observation later on. They did not, however, participate in the design of the sounds.

\footnotetext{
${ }^{5}$ See the demo at https://www.youtube.com/watch?v=x-nIJ0mYghQ where two aerialists performed a duo aerial hoop routine wearing a full-body suit with markers. Note that markers are avoided on the back of the legs of the performers, as they are needed for sitting and hanging.
} 

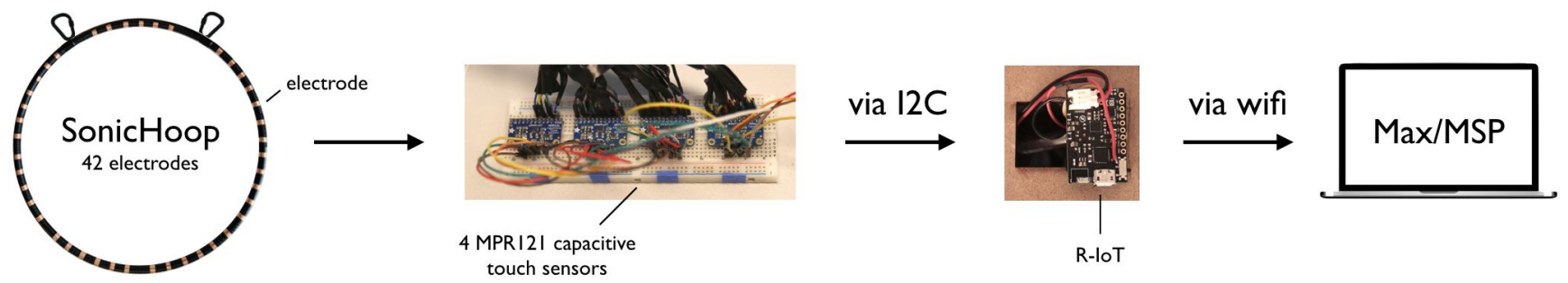

Figure 3: System overview: 4 Arduino MPR121 capacitive touch sensors detect touch events from 42 electrodes covering the whole hoop. The data is gathered by a Bitalino R-IoT and sent to Max/MSP via wifi. SonICHoop works as a standalone device, requiring no extra equipment for the aerialists to wear.

\subsection{Design Choices}

We translated the above-mentioned design goals into four engineering requirements with hardware engineers, and justified our design choices with regular consultation with the two professional aerialists.

3.3.1 Detection Range. Our first engineering requirement is to take various aerial figures into account, which result in different contact areas on the hoop. To do so, we need to know which part of the hoop is used. We analyzed common aerial hoop figures (Figure $2 \mathrm{~b}$ ) and found that, first, all surface areas of the hoop are used depending on the figures and, second, the minimum surface in contact is using the palm of the hand, roughly $8 \mathrm{~cm}$. Therefore, the detection should cover the whole hoop and should identify contact points as small as $8 \mathrm{~cm}$ wide. We rule out depth cameras such as Microsoft Kinect as the constant spinning of the hoop would incur occlusion, making tracking unstable and inconsistent.

3.3.2 Equipment-Free. We want the aerialist to be equipment-free, i.e. to avoid wearables and wires. Many studies that we reviewed in related work (e.g. [3, 30, 84]) require users to wear tracking systems, some of which are quite cumbersome for physical activities. Given the dance-like characteristics of the aerial hoop figures, which involve rotations, spins and drops as well as constant contact between the body and the hoop, it would be uncomfortable and inconvenient for the aerialist to wear something on his/her body. We therefore rule out IMU-suit approaches and aim for an equipment-free design solution.

3.3.3 Minimum Changes to the Hoop. After ruling out depth cameras and motion capture systems using IR markers, we turn to electronics that enable sensing on the hoop. Here the aerial hoop should stay as close to its original status as possible, including minimum changes to its physical construction and its surface area. Depending on the material used, steel or aluminum, the working load limit of an aerial hoop is roughly $200 \mathrm{~kg}$, allowing one to three aerialists to perform on it. We want to preserve its original physical property as much as possible. Similarly, we want to avoid noticeable changes to the hoop surface since it is in direct contact with the aerialist's body. Any changes in material would alter the performer's perception.

3.3.4 Durable and Inexpensive Materials. Finally, the electronics of choice should be able to endure pressure, at least one person's weight. Similarly, it should support figure transitions that involve sudden weight shifting, such as a drop from the top part to the bottom part of the hoop. Given the large surface area that needs to be covered $(85-110 \mathrm{~cm}$ diameter depending on the hoop), we also consider the cost of the sensors. We considered using Force Sensitive Resistors (FSR). FSRs are more expensive and less robust. FSRs can provide pressure sensing, but we did not find it necessary. Finally, we considered mutual- and self-capacitance sensing. Mutual capacitance is more robust to noise and provides a higher resolution, but is more complex, requiring an intersecting matrix of electrodes. Due to the unique size and dimension of the hoop, we found selfcapacitance to be the most appropriate. Self-capacitance is often used for simple buttons and sliders. It works by measuring how long it takes to charge or discharge an electrode. An object such as a finger changes the charge/discharge times, as it adds capacitance. We chose widely commercially available Arduino MPR121 (NXP) sensing chips, which can sense 12-electrodes simultaneously to construct the SonicHoop prototype.

\section{SONICHOOP PROTOTYPE}

In this section, we demonstrate the SonIcHoop prototype, hardware and software, based on our design considerations. Figure 3 provides an overview of the system architecture.

\subsection{Hardware}

We bought a $90 \mathrm{~cm}$ (diameter) 2-point aerial hoop along with hanging straps, carabiners and swivels from firetoys ${ }^{6}$. The aerial hoop weighs $6 \mathrm{~kg}$ and can support up to $300 \mathrm{~kg}$. Since the aerial hoop is made of steel that is naturally conductive, we first wrapped the entire hoop with insulating tape to prevent false detection. After considering the circumference of the hoop $(282.7 \mathrm{~cm})$ and detection range that should identify the palm of the hand (roughly $8 \mathrm{~cm}$ ), we came up with a resolution of 42 electrodes using copper tape (Figure $4 \mathrm{a}$ ). Each electrode has a width of $1.5 \mathrm{~cm}$ and a length of $5 \mathrm{~cm}$, covering $2 / 3$ of the hoop surface from inward, and is placed at $4.5 \mathrm{~cm}$ intervals (except those next to the two carabiners) (Figure 4c), so that wherever the hand is placed, there is always at least one electrode activated.

These electrodes are connected to four MPR121 capacitive touch sensors (Figure $4 \mathrm{~b}$ ), each of which supports 12 inputs, using ultra

\footnotetext{
${ }^{6}$ https://www.firetoys.com
} 


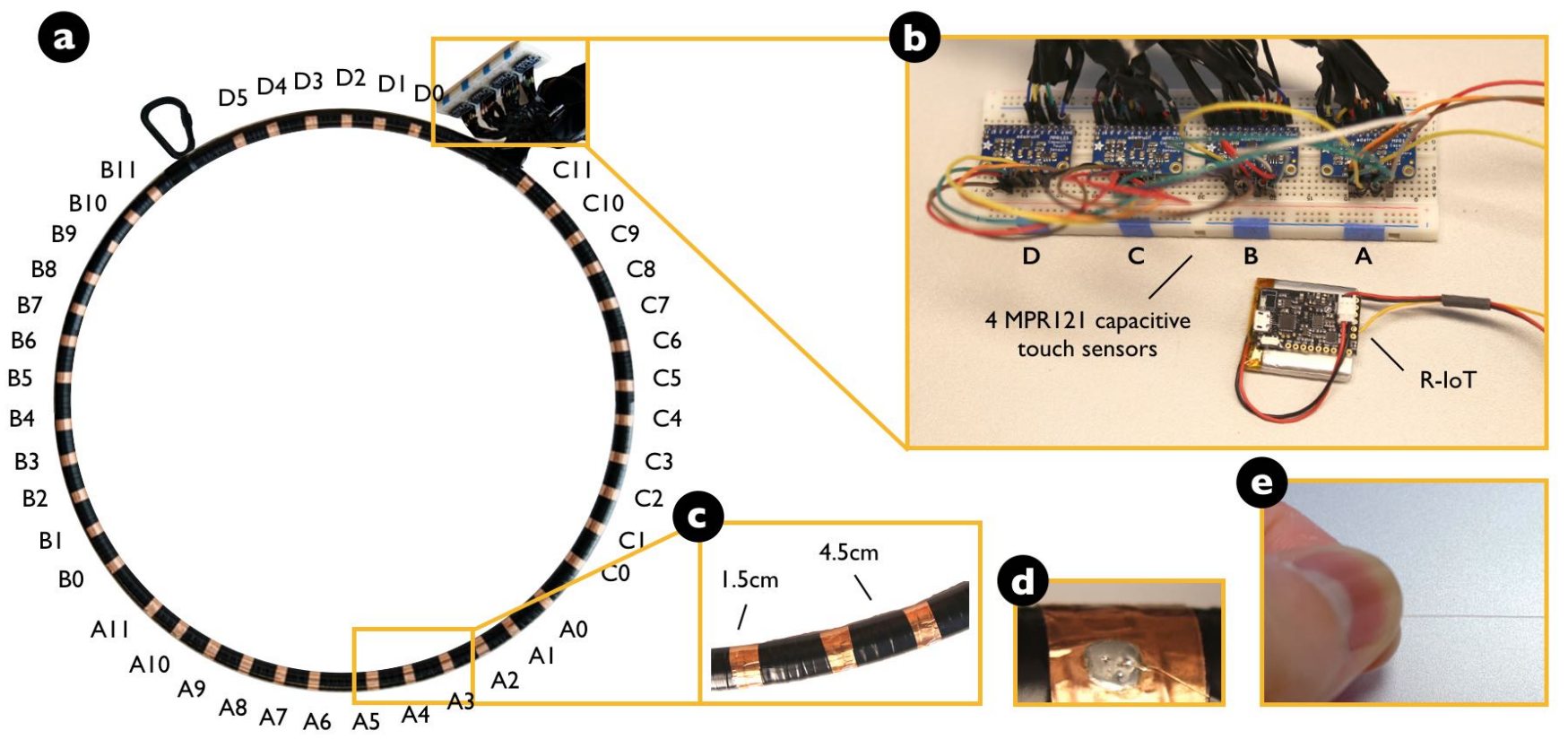

Figure 4: Hardware: (a) 42 electrodes on SonICHoop using (b) four MPR121 capacitive touch sensors and a Bitalino R-IoT. (c) Electrodes are $1.5 \mathrm{~cm}$ wide and $4.5 \mathrm{~cm}$ apart, and are connected to Arduino sensors using (d-e) ultra thin copper wires (0.1mm).

thin copper wire $(0.1 \mathrm{~mm})$ (Figure $4 \mathrm{e})$. We chose this wire to minimize the noticeable changes on the hoop. The ultra thin copper wires are enamelled so we used a cigarette lighter and sandpaper to remove coating at each end. We soldered the electrodes directly onto one end (Figure 4d) and soldered a pin to the other end, which was connected to an MPR121 via a breadboard (Figure $4 \mathrm{~b}$ ). The breadboard is placed between electrode $D 0$ and $C 11$, resulting in the longest wire roughly $160 \mathrm{~cm}$ long (from A11) and the shortest wire roughly $30 \mathrm{~cm}$ long (from $D 0$ and $C 11$ ). We built a I2C communication protocol between Arduino sensors and a Bitalino R-IoT ${ }^{7}$, which streams data to a custom-made Max/MSP program at a rate of 15 milliseconds. We laser cut a wooden box (Figure $1 \mathrm{~b}$ ) to embed all the electronics and covered the hoop with another layer of insulating tape to protect the electrodes (Figure 1a).

SonicHoop works as a standalone device, requiring no extra equipment for the aerialists to wear. The texture of the insulating tape is similar to that used to wrap the cold and slippery surface of a regular aerial hoop. The ultra thin copper wires are covered by two layers of tape so that they should resist pressure and sudden weight shifting. Note that our initial design was to use 7 flex PCBs, each with 6 electrodes, to provide touch sensing (see supplementary material for its design). We did not receive the material due to the impact of Covid-19. Our technical evaluation and user evaluation are therefore conducted using the current version, which costs approximately 60 euros, in addition to the hoop.

\footnotetext{
${ }^{7}$ https://ismm.ircam.fr/riot/
}

\subsection{Software}

We implemented a custom-made program in Cycling'74 Max/MSP to receive and process sensor data, map it to sound as well as record touch data and audio (Figure 5). The program receives the raw values of sensor data, which is a list of 42 floats with the standby value as 34 every 15 milliseconds, and decides on when a touch is triggered after applying two filters. The first filter is a moving average filter (on the previous 10 data points) to smooth out the noisy raw data. The second filter is a step detection filter to detect abrupt changes in noisy time series data. We used an online version that compares the new data point to the median of the previous 20 data points and defined the threshold as 0.2 . That is to say that if the difference between the new data point and the median of the previous 20 points is greater than 0.2 , we consider it as a touch event (touch 1 or release 0 ). This approach has tradeoffs: it maximally eliminates false detection, but it sacrifices latency since applying two filters consumes some time before we can pass the data to generate sound. We evaluate and further discuss this latency in the later section. Both filters are implemented using Pipo [76], a plugin API for data stream processing in Max/MSP.

We also created a visualization tool that provides real-time presentation of the electrodes' status (Figure 6). When not triggered, electrodes are visualized in blue. When triggered, electrodes are visualized in red. The centroid of activated electrodes is also computed for sonification, which will be described in the next section.

\section{SONIFICATION STRATEGY}

The details of the sonification clearly matter, as they potentially cause large differences in the aerialist's movements, perception, and 


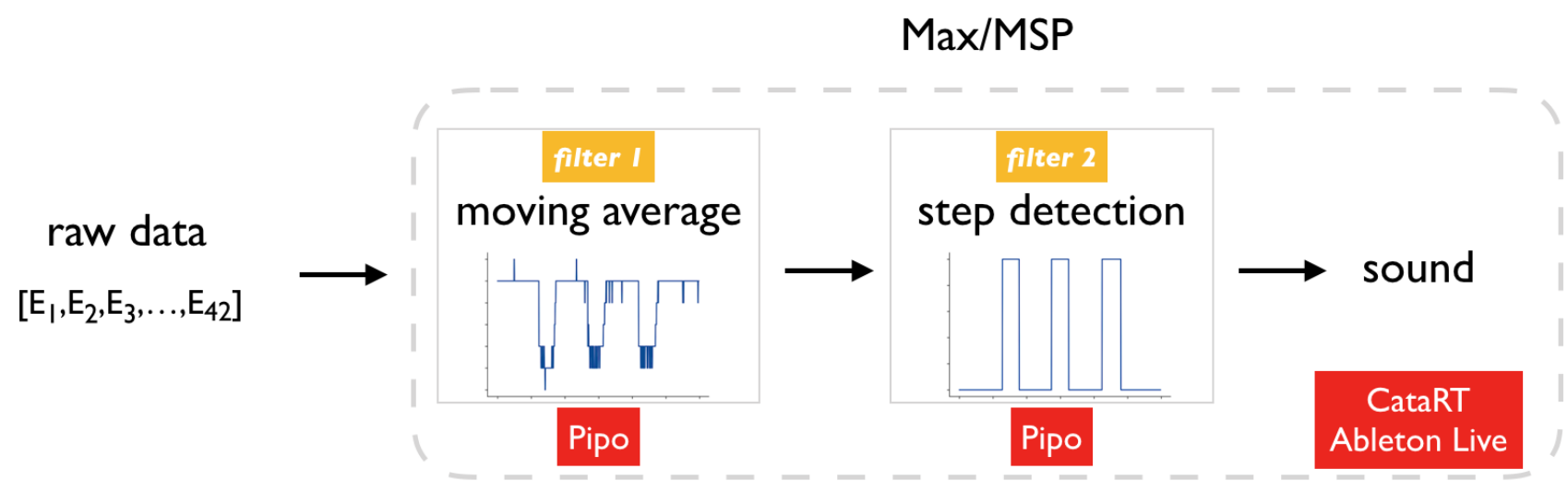

Figure 5: Software: Max/MSP receives the raw touch data, a list of 42 floats every 15 milliseconds, smooths it using a moving average filter and detects touch events (touch or release) using a step detection process, and generates sound accordingly. The two filters are implemented using Pipo and sound is generated using CataRT and Ableton Live.

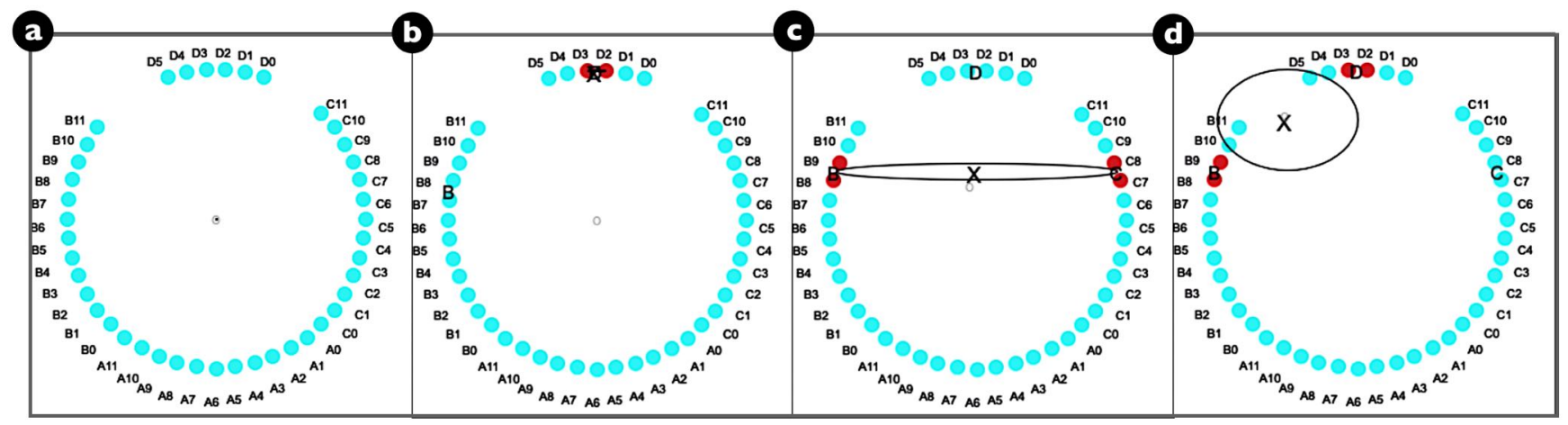

Figure 6: Visualization of the electrodes' status in real time: (a) default status with no touch events; (b)-(d): touch events are detected and the centroid among touch points is computed for sonification.

choreographic processes. Our goal is to go beyond simple sounds and mapping strategies as often used in interactive sonification in sports and instead provide a musical aesthetic. Therefore, we intentionally avoided mapping touch to low-level parameters such as a specific sound or sound property such as pitch. We developed three sonification strategies that vary the character of the sound, choice of sequence and layering of loops. Each sound design is distinct from the others in terms of musical style and composition strategy. To facilitate reading, we call them ambient, lounge and electro dance, according to their musical style.

\subsection{Ambient}

The first sonification strategy explores how to let performers create pleasing, ambient sounds from nature. The goal here is not to create a particular musical style, but rather to let the performer fluidly combine sounds to create a coherent result. We divide the hoop into 4 sections, each of which produces a different type of dense ambient sound corresponding to earth (clay tiles), wind, water and birds (Figure 7a). This asymmetric design is inspired by how a sequence of figures is composed (see design considerations): an aerialist often starts from the floor, using the bottom part of the hoop to go up, the side parts for a series of figures and the top part to go even higher.

In each section, the centroid of activated electrodes is mapped to a dense collection of short sound samples (grains), ordered by their sonic character. For instance, for the wind section, touching on the bottom (e.g. $\mathrm{CO}$ ) will play layered soft sounds of weak wind, and moving upwards will gradually play louder, stronger wind sounds with more high-frequency content. Additionally, the spread of the touch activation for each section is mapped to the range around the centroid from which sound grains are selected. For example, touching the top section with one hand will play a continuous texture of a specific bird sound character, while touching with both hands at the extremities of the section will play a large variety of different bird sounds.

The sound collections are recordings of natural phenomena and are constituted into a corpus by timbral analysis in the CataRT system [77] for Max/MSP. This approach of corpus-based concatenative synthesis allows us to use the rich and nuanced sound world 


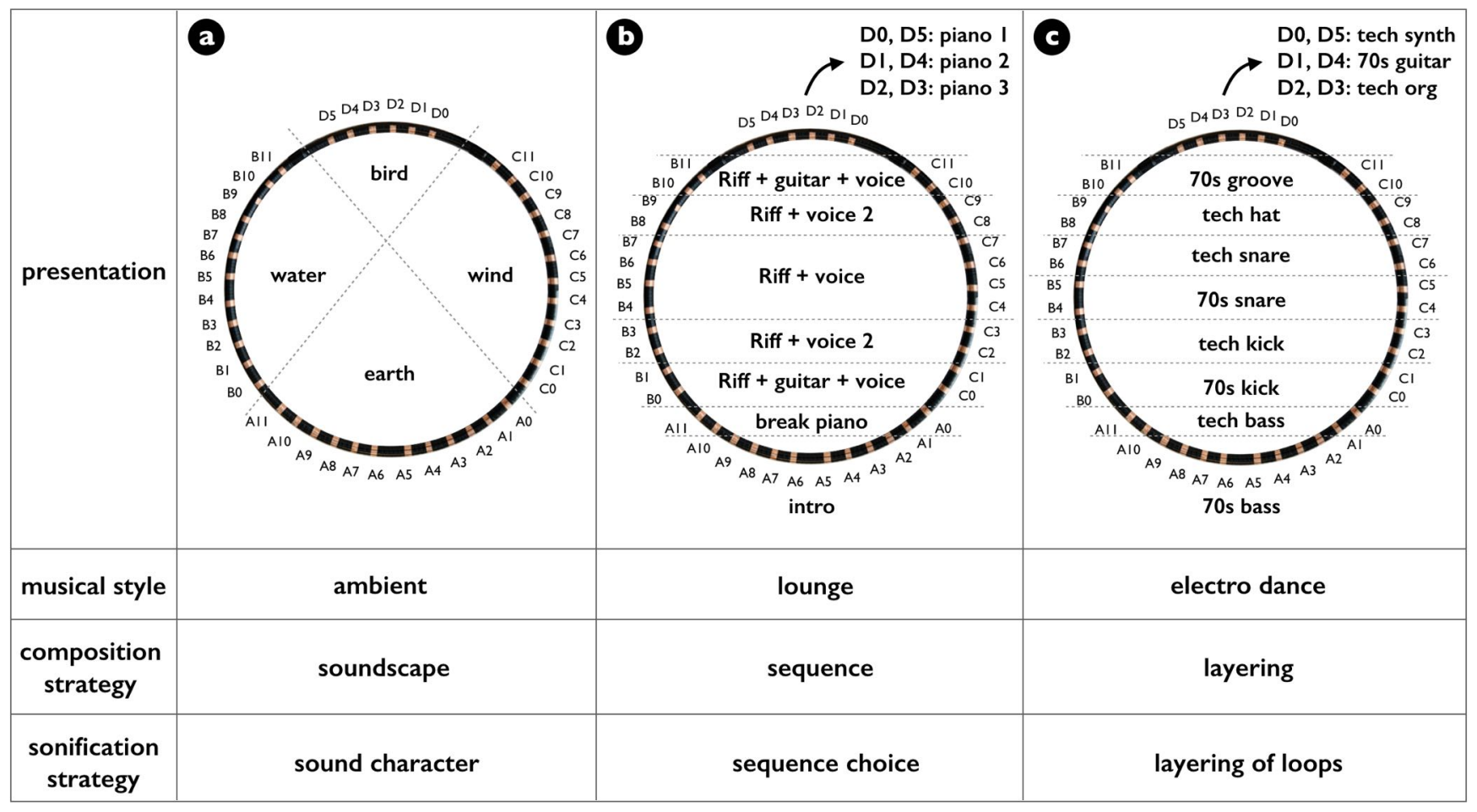

Figure 7: Three sonification strategies varying in presentation, musical style and composition strategy: (a) ambient; (b) musical and (c) electro dance.

of real natural environments, and to still be able to create continuous sonic evolution by sound selection via audio descriptors, without having to resort to audio manipulation.

\subsection{Lounge}

The second sonification strategy explores how to let performers dynamically modify the expressive quality of a particular musical style, in this case, lounge music. The goal is to let performers dynamically control the orchestration of the piece by moving up and down the hoop. We divide the hoop into 10 vertical zones that switch to the music segment assigned to it (Figure 7b). This symmetric design rationale (also used for electro dance, described in the next subsection) is based on the fact that for each aerial hoop figure, one can practice both on the left side and right side. This creates a mirroring representation, similar to other dance figures.

We chose a set of 11 segments of a music track in a jazzy lounge house style, mapped 10 of them to the 10 zones of vertical space, and the last one for the fadeout effect to add an ending to the piece. The switch occurs on the next $16^{\text {th }}$ note, jumping to the same metric position in the target loop, thus maintaining rhythmic consistency. The segments are arranged in a rough order of musical buildup, with calm parts at the bottom (the intro just with bass, then a solo piano part), and gradually denser parts of the arrangement (bass and different synthetic choir voice and guitar parts) towards the top. At the top section, we placed three different parts with piano, which have very bright timbres.

\subsection{Electro Dance}

The third sonification strategy explores how to let performers combine different instruments based on their position around the hoop. Similar to the symmetric presentation of lounge, described above, we divide the hoop into 11 vertical zones and allows to layer $133 \mathrm{bpm}$ loops in an energetic electronic dance music style (Figure 7c). The loops contain one type of instrument each and are designed to be layered to build up a composition. Loop start and loop stop are rhythmically synchronous to the already running loops. A similar setup can be seen in Entrain [78], a distributed and collective musical instrument that synchronizes eight layers of sequences.

Both lounge and electro dance sonification are created using Ableton Live ${ }^{8}$ which communicates with Max/MSP back and forth. All three sonifications also provide for a two-second special musical fade-out effect when the aerialist lets go of the hoop at the end of the performance.

\section{TECHNICAL EVALUATION}

In this section, we evaluate the touch-to-sound latency and sensitivity to different textiles of SonicHoop.

\footnotetext{
${ }^{8}$ https://www.ableton.com/en/live/
} 


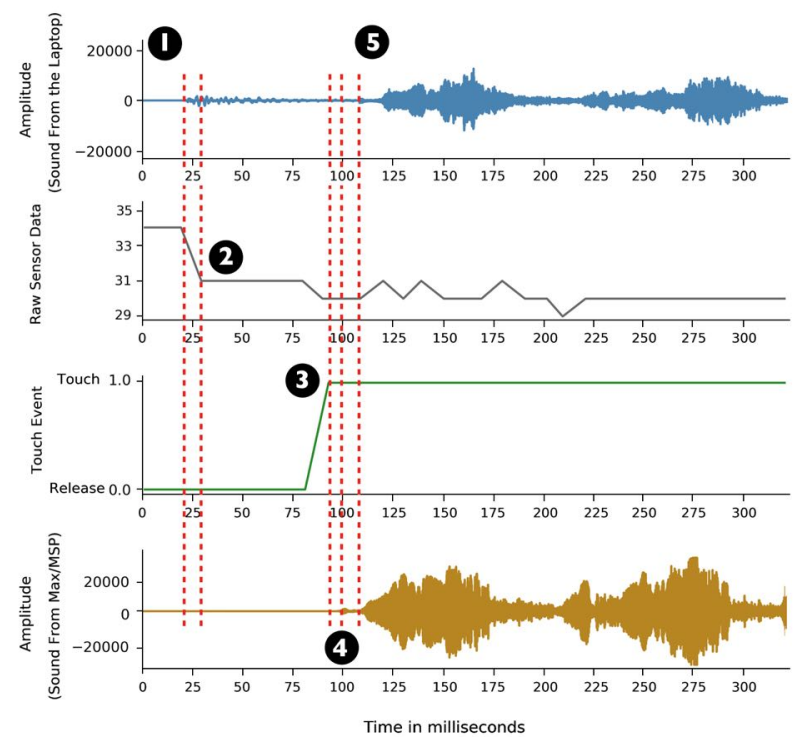

Figure 8: Touch-to-sound latency of SonicHoop: (1) touch on the hoop; (2) Max/MSP receives the raw sensor data; (3) a touch event is triggered after applying 2 filters; (4) a sound is generated accordingly in Max/MSP and (5) the sound is output from the laptop's speakers. (1)-(2): $10 \mathrm{~ms},(2)-(3): 82 \mathrm{~ms}$, (3)-(4): $8 \mathrm{~ms},(4)-(5): 10 \mathrm{~ms}$. Total latency (1)-(5): $110 \mathrm{~ms}$.

\subsection{Touch to Sound Latency}

First we evaluate the latency between body contact on SonicHoop and generated auditory feedback. This is important as any significant delays in feedback would negatively impact the actionperception process. Using the external stereo digital recorder paradigm [87], we measured the total system latency (Figure 8 (1)-(5)) between a touch event (picked up by a contact microphone) and the start of the output sound as $110 \mathrm{~ms}$, below the just noticeable threshold $200 \mathrm{~ms}$ [40]. Additionally, we recorded the internal processing steps (2)-(4) in order to estimate their respective contribution to the total latency. More specifically, it takes approximately $10 \mathrm{~ms}$ for Max/MSP to receive the raw sensor data (step (1)-(2)), $82 \mathrm{~ms}$ to detect the touch event using the two filters (step (2)-(3)), $8 \mathrm{~ms}$ to generate sound in Max/MSP once a touch event is considered true (step (3)-(4)) and finally $10 \mathrm{~ms}$ to output sound from the laptop's speaker (step (4)-(5)). The audio setting of the laptop (a MacBook Pro with a $2.5 \mathrm{GHz}$ processor and 16 GB RAM) was I/O vector size 128 and signal vector size 64 .

The most time-consuming process is to detect a touch event using the two filters ( $82 \mathrm{~ms}$ out of $110 \mathrm{~ms}$ ). As stated earlier in software description, this is a conscious choice to maximally avoid false positive triggering while guaranteeing high sensitivity. The sampled data is noisy due to the noise from capacitive touch sensors as well as from wifi streaming. Given the nature of auditory feedback (a sound might be generated when not touched), we think that this is a worthwhile trade-off. Other filtering techniques could be applied to ensure both high detection accuracy and shorter time cost.

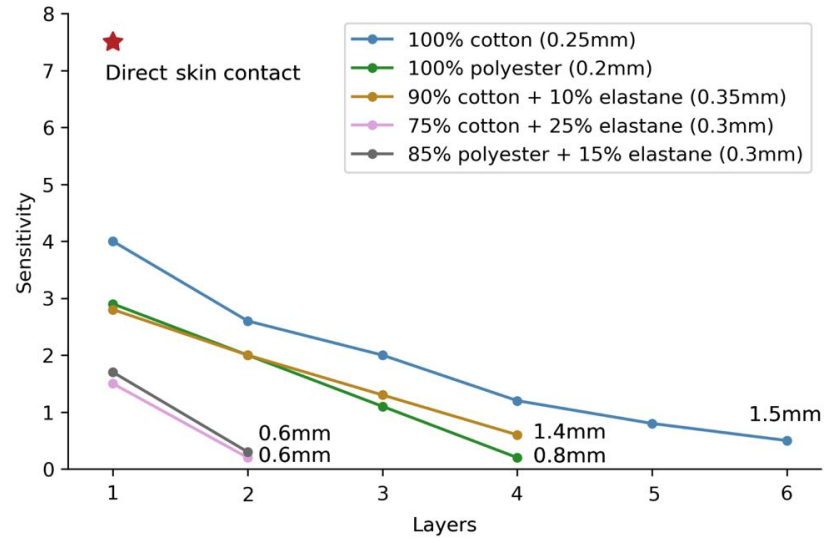

Figure 9: Sensitivity to 5 different textiles: SonicHoop can detect touch events when applying up to $1.5 \mathrm{~mm}$ of $100 \%$ cotton, up to $0.8 \mathrm{~mm}$ of $100 \%$ polyester, up to $1.4 \mathrm{~mm}$ of $90 \%$ cotton with $10 \%$ elastane, and up to $0.6 \mathrm{~mm}$ of $75 \%$ cotton with $25 \%$ elastane and $85 \%$ polyester with $15 \%$ elastane. Sensitivity is calculated using the difference between sensor standby raw value (34) and the decreased value when touch is applied, e.g. direct skin contact $=\mathbf{2 6 . 5}$, therefore sensitivity $=7.5$.

\subsection{Sensitivity to Different Textiles}

We also evaluate SonicHoop's sensitivity to different textiles as the aerialist wears sports clothes during practice sessions or costumes for performances. The most direct skin contact comes from the palm of the hand, feet and back legs if shorts are chosen over long leggings. Therefore, it is important to know to what extent SonicHoop can detect touch events, when extra layers of textiles are worn.

We compared direct contact with skin, which results in the highest sensitivity, with 5 different textiles: $100 \%$ cotton, $100 \%$ polyester, $90 \%$ cotton with $10 \%$ elastane, $75 \%$ cotton with $25 \%$ elastane and $85 \%$ polyester with $15 \%$ elastane (Figure 9 ). The last three are very common compositions of sports clothes that provide elasticity and strength.

The findings indicate that cotton has the highest sensitivity among the 5 textiles (up to $1.5 \mathrm{~mm}$ ), since cotton is usually the most capable textile to absorb humidity in air to enable some conductivity. Polyester is less conductive than cotton. It can enable touch detection on SonicHoop up to $0.8 \mathrm{~mm}$ thick. The more elastane is added, the less conductive the textile gets $(1.4 \mathrm{~mm}$ for $90 \%$ cotton with $10 \%$ elastane, $0.6 \mathrm{~mm}$ for $75 \%$ cotton with $25 \%$ elastane or $85 \%$ polyester with $15 \%$ elastane). Based on this evaluation, we kept our initial threshold to distinguish touch events as 0.2 .

\section{STRUCTURED OBSERVATION}

To understand how auditory feedback, particularly how different sonification strategies, influence aerialist's movements and choreographic processes, we conducted a structured observation [25, 39, 48] with two professional aerial hoop performers. Structured observation is a type of quasi-experiment [16] that combines controlled 
conditions with empirical observations to enhance ecological validity. Participants are asked to perform explicitly comparable tasks and provide detailed, qualitative feedback about each. Our goals here are to first, observe how interactive sonification affects aerialist's perception and movements with respect to the artistic and sportive aspects of aerial hoop practices, and second, to ask them to compare and reflect upon the various advantages and disadvantages of different sonification strategies, offering us deeper insights and implications for design.

\subsection{Participants}

We recruited two professional aerial hoop performers/teachers, both female. P1 (41 years old), was initially trained as a jazz dancer; has more than 20 years experience in circus performances; teaches aerial hoop, pole dance and Pilates on a daily basis. P2 (33 years old), was trained in gymnastics since age 5 ; has 10 years experience in teaching aerial hoop, pole dance, contortion and yoga on a daily basis. Both participants provided informed consent and agreed to the recording of the session and publication of the results. Each received $50 €$ (due to the physical demand of the study) for their participation.

\subsection{Setup}

The study took place in the dance studio where both participants teach aerial hoop. Three experimenters and one participant were present at the same time in a room of 100 square meters, all (except the participant) wearing masks, respecting social distancing and governmental regulations. SoNicHoop was hung from the $2.8 \mathrm{~m}$ high ceiling with the bottom part $1.4 \mathrm{~m}$ from the floor. The same laptop was used and a loudspeaker was placed $1.5 \mathrm{~m}$ away (Figure 10a). SonicHoop as well as the two yoga mats that were placed below SonicHoop were disinfected after each study.

\subsection{Procedure}

The study takes approximately 75 minutes and includes the following steps (Figure 10c):

Familiarization: Participants are introduced to the concept of SonicHoop and are given 5 to 10 minutes to familiarize themselves with SonICHoop using a 1-minute predefined sequence of figures. This sequence has 6 intermediate-level figures that can be performed by professionals effortlessly, therefore it does not involve movement learning. The goal of this phase is twofold: first, participants can familiarize themselves with SonIcHoop's physical construction, including size and surface texture; second, participants can practice and memorize the sequence for the controlled practice that comes next.

Controlled practice: Participants practice the same sequence using three sound conditions (P1: electro dance, lounge, ambient; P2: lounge, ambient, electro dance). Before practicing, they are encouraged to touch different parts of SonicHoop to get some ideas about the sound design. After practicing, they fill out a 5point Likert-style questionnaire and use a talk aloud protocol to describe their experience performing the predefined figures in this condition.

Exploration: Participants explore freely using each sonification strategy for 5 to 10 minutes.
Semi-structured interview: Participants fill out another Likertstyle questionnaire, and use a talk aloud protocol to compare the different sonification strategies and describe their overall experiences.

\subsection{Data collection and analysis}

We collected video, audio, questionnaires and hand-written notes. We analyzed results using a mixed-approach thematic analysis [12], with top-down themes, the three sonification strategies (ambient, lounge and electro dance), as well as bottom-up themes that emerged during the coding process.

\subsection{Results}

Overall, participants find that auditory feedback, regardless of sonification strategy, provides useful information about their movements, thus improving perceived movement quality (Figure 11): "since the sound is corresponding to your movement, you are obliged to pay attention to the characteristics of a figure, for example duration, which you don't usually pay attention to" (P1); "it definitely improves movement quality since we get to hear how the figures sound like" (P2). However, different sound designs affect how they interpret figures, both visually and acoustically, of choreographing, as well as their attention differently.

7.5.1 Ambient. The ambient sonification strategy has no independent rhythm of its own, which made it easy for participants to improvise and explore figures in a way that is completely opposite to their normal practices. As mentioned before, an aerial hoop piece is usually choreographed according to the characteristics of the chosen music, which means that the music drives the aerialist: "Usually you need to translate the music into your movement"(P1). The ambient sonification strategy caused the aerialists to fundamentally change how they thought about choreographing and performing, and was easily their preferred sonification approach. P1 said: "With a regular aerial hoop, we always think about which figures to do and which figures follow which other figures. But here I was not thinking about figures at all, but more how to orient myself on the hoop, where to put my hands, my feet etc. We leave the zone of using techniques but more searching for new expressions. It's a different thinking process, which is super nice and more poetic. Also it's the sound of nature, it feels like a journey". P2 said: "The sound is very relaxing and almost meditative. So I pay more attention to the sound. Sometimes I deliberately did not touch the top part because I know that it's the sound of the birds, and at the moment it didn't fit into the story. So I completely changed my movements and consciously made choices for which sound to use to tell the story".

7.5.2 Lounge. The lounge sonification strategy is the closest to music they would choose for an aerial hoop piece. P2 said: "It really makes me feel like dancing, stretching and having fluidity". However, the continuity of music - each movement triggers different segments of the music track that continue afterwards - might disrupt free expression. P1 said: "I'd like to have some silent periods, for example, if I stay in a position for a while, maybe it could create some silence? You saw that sometimes I was looking for movements that allow me to not touch any part of the hoop (such as the straps on top) so that I could hang without getting down on the floor, but do not 

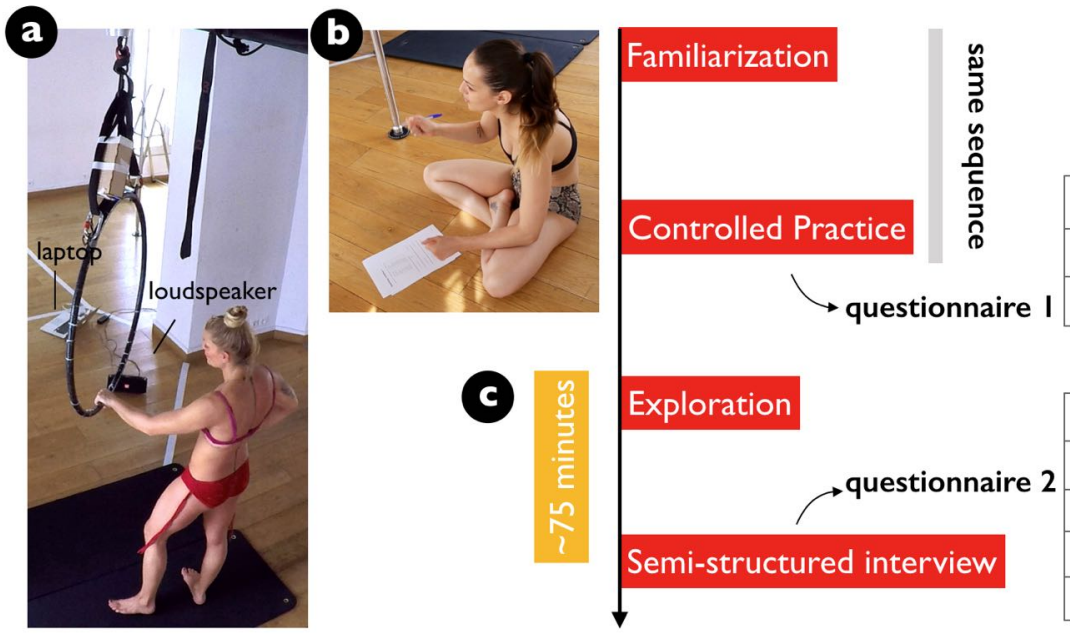

I found that sound helps concentration

I found that sound helps improve my movement quality

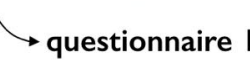

It is easy to understand the relationship between my movement and sound

○

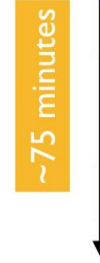

\section{Exploration}

I found SonicHoop interesting as a concept

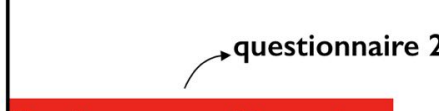

Semi-structured interview

I found SonicHoop useful to explore figures for choreography

I found SonicHoop useful for my personal artistic expression

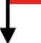

I would be interested in using SonicHoop in my class

I would be interested in using SonicHoop to explore new artistic forms

Figure 10: Experiment setup: (a) SoNICHoop is hung from the ceiling $2.8 \mathrm{~m}$ high and a loudspeaker is placed $1.5 \mathrm{~m}$ away $\odot$ Selma Marin; (b) P2 talks aloud while filling out a questionnaire $\odot$ Momo Power; (c) experiment procedure.

\begin{tabular}{|c|c|c|c|c|c|c|c|c|}
\hline \multirow{2}{*}{ Questionnaire Questions } & \multicolumn{2}{|c|}{ ambient } & \multicolumn{2}{|c|}{ lounge } & \multicolumn{2}{|c|}{ electro dance } & \multicolumn{2}{|c|}{ overall } \\
\hline & $\mathrm{PI}$ & $\mathrm{P} 2$ & $\mathrm{PI}$ & P2 & $\mathrm{PI}$ & P2 & $\mathrm{PI}$ & $\mathrm{P} 2$ \\
\hline I found that sound helps concentration & 5 & 5 & 5 & 3 & 4 & 4 & & \\
\hline I found that sound helps improve my movement quality & 5 & 5 & 5 & 5 & 5 & 5 & & \\
\hline It is easy to understand the relationship between my movement and sound & 5 & 4 & 4 & 4 & 3 & 4 & & \\
\hline I found SonicHoop interesting as a concept & & & & & & & 5 & 5 \\
\hline I found SonicHoop useful to explore figures for choreography & & & & & & & 5 & 5 \\
\hline I found SonicHoop useful for my personal artistic expression & & & & & & & 5 & 5 \\
\hline I would be interested in using SonicHoop in my class & & & & & & & 2 & 3 \\
\hline I would be interested in using SonicHoop to explore new artistic forms & & & & & & & 5 & 5 \\
\hline
\end{tabular}

Figure 11: Descriptive statistics from questionnaire 1 for each sonification strategy (in yellow) and from questionnaire 2 for overall experience (in red).

generate sound. Otherwise if I want to have silent moments, I have to completely let go and get down on the floor. I think this would be very interesting to have this 'suspension' moment and then continue".

7.5.3 Electro dance. The aerialists understood the electro dance sonification strategy less well than the other two, in terms of the relationship between their movements and the sound (Figure 11). This was also their least preferred sonification strategy, as the rapid music tempo (133 bpm) forced them to follow the rhythm, with a corresponding sense of loss of control. P1 said: "In fact, I can stay with the rhythm since it already has one, but without knowing that it's me who creates sounds, it's difficult. It feels like the sound just keeps going, and I can put myself in there (follow the rhythm), but I don't have a sense that it's me who's in control". P2 said: "This sound is perhaps the best for a concert performance or when it's more dynamic. It's a different type of dance where you would adapt your movements more to the rhythm. For something more rhythmic, it's definitely the performer who needs to adapt to the rhythm, rather than for example with the environmental sound (ambient condition), we can choose how we'd like to move. Since this is something interactive, I guess it's more pleasant to have something that you're in control of". P1 also found it difficult to differentiate all 11 sounds: "There are lots of different sounds but many sounds are difficult to distinguish, such as bom bom and ding ding, you see. I have a hard time knowing if I'm in contact with something or not".

7.5.4 New expressions. Both participants agree that SonicHoop is an interesting and useful tool for exploring choreographic figures, 
as well as for personal artistic expression. P1 said:"It would be super interesting because you know usually we try a sequence of figures and see if it works. Here by listening to the sound, you know whether this figure would work or rather break the music after this figure, so it's really helpful. When you dance to a piece of music, you don't necessarily realize that". P2 said:"It would be something super interesting not just for myself to look for new expressions, but also for those who are not necessarily trained in music, you know. For instance, some people who are not very good at putting themselves into music, or a bit shy expressing themselves artistically, I think this would really help them".

7.5.5 Pedagogy. When asked whether they would be interested in using SonicHoop in their class, both participants considered its potential benefits, but also voiced concerns. P1 said: "I think it'd be interesting as a pedagogy tool. For example, as I said earlier that it'll be great to use it to communicate the duration of figures. However, it'd also be difficult as I'm thinking about a collective class with for example 10 students, each of whom has one SonicHoop. Then each student needs a headphone so that they're not disturbing each other with sounds. It could be possible to use it for a class of choreography for those who already have the techniques and would like to go further. For beginners, I think there are just too many things. For me, I think we really need to start with techniques, and then we explore with SonicHoop. I see this more of a performance tool for shows and spectacles". P2 said: "It's not that I don't think it's interesting but I think it's less accessible for the students, especially those who're still developing their skills. There is just too much information for them, already the movements, and now if we add something musical, it would make it even harder. It would be interesting for a class but it depends on which students and whether they're already comfortable on the hoop. We can certainly use this to communicate how to express a figure, how long do we need to stay in the figure, how do we transition into a different figure etc. For instance, you wouldn't do the same thing when the music is fast or when the music is slow. So it helps training variability? And in the class, it might also help students to feel closer to the hoop and find their own way of expressing. Or even for professors to find new ways of using the same instrument and expressing themselves".

7.5.6 Aerial hoop performances. Both participants said that they would be interested in an interactive aerial hoop performance. However, they were also concerned by how to communicate the auditory interactivity to the audience. P1 said: "I think it's also very important to make the public understand that it's the artist who is creating the sound". P2 said: "I think it's super interesting to explore new forms for performances because it's something super novel super cool. But I think it might be useful to, I don't know, add visual information for the audience to understand that it's the artist who is composing the music. I say this because you know, for a public show if you do a split on the aerial hoop, the audience would be like wow amazing. But if you do something less visually impressive but in fact super difficult and technical, there is no response from the audience. So in fact it might be useful to use sound, I don't know, to communicate such information?".

7.5.7 More nuanced sound design. We also asked participants about their overall experience with the sound design and whether more nuanced design strategies might be possible, for instance, adding special sound effects using the rotation speed of the hoop, captured by the R-IoT sensor. Both participants mentioned that all three sonification strategies are interesting and would be useful for different occasions. P2 said: "All three work very well and I think it depends on the occasion, whether it's a public concert that requires more dynamic music or something more artistic and slow. It's like you wouldn't wear the same costume given different music and occasion". P1 said: "It could be interesting to use the rotation speed to add special sound effects but also maybe dangerous. There I'm really imagining myself in a show and you know it's difficult to always have the same rotation speed, so it means that the sound will change as well. You know the spinning depends on if you have a short hang or a long hang etc. so it could be dangerous. There might also be a conflict between my own rhythm on the hoop such as certain movements and certain transitions, which consequently control the spinning speed itself. So even though it could be interesting to use rotation speed to provide certain sound effects, we need to be careful".

\section{DISCUSSION}

We evaluated SonicHoop with respect to its touch-to-sound latency and its sensitivity to different textiles, and identified the most promising candidates for interacting with a capacitive-touch based aerial hoop. We also conducted a qualitative structured observation that compared different sonification strategies and offered insights as to how each influences the practice and performance of two professional aerialists. We found that all three types of interactive sonification improve movement quality and can be used in different occasions. We also found that ambient sounds, with no independent rhythm, offer performers the greatest freedom of expression, whereas music with repeating loops or large numbers of different sounds might disrupt their freedom of expression. This section discusses SonicHoop from different perspectives: as an aerial hoop training tool, as a digital musical instrument, and as a creative object. We then address limitations of this work and directions for future research.

\subsection{SonicHoop as an Aerial Hoop Training Tool}

Participants receive intrinsic feedback when performing on the aerial hoop according to its rotation speed, which body parts are in contact with which parts of the hoop, etc. They also receive extrinsic auditory feedback, which provides additional information about the quality of their movements, and keeps them aware of aspects of each figure that are often left out. These characteristics, e.g. duration of a figure, transitions between figures and the expression of a figure, along with the teacher's intentions and perceptions can then be further communicated to students in a pedagogical setting. However, the participants also mentioned that SonicHoop would be less accessible for beginners since they are still developing their skills, and implies that adding auditory feedback to movement might make it harder. This echoes the limited literature on the effects of auditory feedback on movement learning: the effectiveness of interactive sonification to facilitate learning has been mostly explored for simple tasks [81] and it is difficult to generalize to more complex movements, such as those involved in aerial hoop. One study, with a limited statistical analysis, postulates that auditory 
feedback is beneficial for expert users but not novice users [34]. The effectiveness of auditory feedback also depends upon one's musical experience and abilities [22]. This suggests that we would need to modify the features and aesthetics of SonIcHoop sound design, such as using low-level parameters and simple movement-to-sound mappings, if we are to cater to beginners' needs.

\subsection{SonicHoop as a Digital Musical Instrument}

We see the potential of SonicHoop as a digital musical instrument (DMI) that aerialists play with their bodies. During the study, participants also provided valuable insights as to how the interplay of sound and movement affects the creative process, similar to other musical instruments. Within the context of DMIs, SonIcHoop is also a unique case where sound production comes from an artistic discipline - here, playing the instrument is a sport in its own right. One specific characteristic of SonicHoop is that no absolute "correct sound" exists for particular figures - see the supplementary material for examples of sounds generated by the two participants when they used the same sequence. First, each aerial hoop has a certain size; this version is $90 \mathrm{~cm}$ in diameter. This means that, given the size of the performer, the same figure will sound differently as the performer ends up in a slightly different position on the hoop. Second, even the same performer might end up in a different position, depending on their actions prior to transitioning to a particular figure. One potential solution would be to map the same sound to clusters of activated electrodes with similar compositions. This could be done by collecting extensive data from more aerialists as they perform the same figures using different sequences, and training a machine learning algorithm to recognize these figures. The current version of SoNICHoop serves as a highly individualized musical instrument that emphasizes both sound production and musical expressivity $[18,28]$.

\subsection{SonicHoop as a Creative Object}

In addition to its roles as a training tool and as a digital musical instrument, SonicHoop can also be viewed as a creative object in its own right, one that encourages exploration and novel expression. SonICHoop enhances participants' perceptions of their own movement qualities and, depending upon the sonification strategy, fundamentally alters their choreographic processes. Instead of translating music into movement, these artists search for bodily expressions that they use to actively compose music. This is particularly true of the sounds in the ambient condition, which lack their own rhythm. Here, the music no longer drives the aerialist, but rather the aerialist drives the music. Instead of simply choreographing a sequence of figures, they choreograph with the body, both reacting to and controlling the sound as they perform. This change of perception is similar to creativity support techniques seen in dance and somatic practices [33], such as moving and making strange [44] and defamiliarization [13]: All emphasize "varying our normal movement patterns and processes so that we can unsettle our habitual perceptions of the world and ourselves". We argue that SonICHoop offers a new perspective on an otherwise familiar process, and can further support creativity in artistic sports beyond aerial hoop practice and performance.

\subsection{Limitations and Directions for Future Research}

8.4.1 Number of participants. Our goal was to understand the various roles SonicHoop can play at the intersection between athletic and artistic practice. We were particularly interested in how different sonification strategies affect performance, perception and choreographic processes, which requires highly skilled aerial hoop practitioners. We chose to work with two professional aerialists who are both performers and teachers over an extended period to gain more in-depth understanding and insights. Particularly, structured observation lets both the participants and ourselves explicitly reflect upon and compare the three sonification strategies.

An alternative would have been to work with beginner and intermediate students, however this would require completely different sound designs. Moreover, unlike the professionals, students need to learn each sequence, which could bias the results with respect to the ultimate use of SonicHoop. The use of students would shift the emphasis from choreography and performance to learning, which is another interesting topic for future research. A larger-scale study could also explore different sound designs and sonification strategies. However, we believe that the current study offers useful first step in exploring the role of sonification in activities that combine both athletic and artistic requirements.

8.4.2 Sensing limitations. Even though neither participant experienced feedback delay, textiles clearly have the potential to limit sensing. Our technical evaluation successfully demonstrated SoNIcHoop's sensitivity with five different textiles. However, during the structured observation study, some touch events were missed. This might be because the participants were wearing other compositions or layers of materials that exceed the detection range. As a temporary solution, participants wore crop tops and shorts to maximize skin contact during the structured observation. Future work will test other filters and sensing techniques that allow SoNIcHoop to better handle layers of textiles. Furthermore, we plan to infer figures in real-time from the sensor data using machine learning techniques, which we believe could make the detection more robust to ambiguity, as inference would be aggregated from multiple sensors.

\section{CONCLUSION}

We present SonicHoop, an augmented aerial hoop with capacitive touch sensing and interactive sonification. We describe our design considerations based on aerial hoop practices, the hardware and software of the SonicHoop prototype. We also present three different sonification strategies and describe the results of a structured observation study with two professional aerial hoop performers.

The results indicate that regardless of sound design, interactive sonification provides useful information about the aerialist's movements on the hoop and improves movement quality. Furthermore, depending on the sonification strategy, SoNicHoop changes how aerialists perceive and choreograph: instead of translating music into movement, they search for bodily expressions to compose music. This change of perception can further be cultivated into other creative sportive or artistic activities. 
Future work includes exploring other sensing techniques and sonification strategies, and testing them with more participants of different skill levels. We also plan to investigate interactive sonification in other acrobatic practices such as aerial silks to further explore full-body vertical interaction.

\section{ACKNOWLEDGMENTS}

We thank the participants for their time and insights, the reviewers for their feedback, and our colleagues Antoine Loriette and Jean-Philippe Rivière for their help in conducting the structured observation. This research was partially funded by ELEMENT Enabling Learnability in Embodied Movement Interaction (ANR18-CE33-0002), and by European Research Council (ERC) grants $n^{\circ} 695464$ "ONE: Unified Principles of Interaction” and n 321135 "CREATIV: Creating Co-Adaptive Human-Computer Partnerships".

\section{REFERENCES}

[1] SIGGRAPH 2019. 2020. SIGGRAPH 2019. https://s2019.siggraph.org. Accessed 2020-08-20.

[2] Jan Anlauff, Jeremy R Cooperstock, and Joyce Fung. 2013. Augmented feedback for learning single-legged stance on a slackline. In 2013 International Conference on Virtual Rehabilitation (ICVR). IEEE, 162-163.

[3] Marc Bächlin, Kilian Förster, and Gerhard Tröster. 2009. SwimMaster: A Wearable Assistant for Swimmer. In Proceedings of the 11th International Conference on Ubiquitous Computing (Orlando, Florida, USA) (UbiComp '09). Association for Computing Machinery, New York, NY, USA, 215-224. https://doi.org/10.1145/ 1620545.1620578

[4] Ludovic Baudry, David Leroy, Ràgis Thouvarecq, and Didier Chollet. 2006. Auditory concurrent feedback benefits on the circle performed in gymnastics. fournal of sports sciences 24,2 (2006), 149-156.

[5] Frederic Bevilacqua, Fabrice Guédy, Norbert Schnell, Emmanuel Fléty, and Nicolas Leroy. 2007. Wireless Sensor Interface and Gesture-follower for Music Pedagogy In Proceedings of the 7th International Conference on New Interfaces for Musical Expression (New York, New York) (NIME '07). ACM, New York, NY, USA, 124-129. https://doi.org/10.1145/1279740.1279762

[6] Frédéric Bevilacqua, Norbert Schnell, Nicolas Rasamimanana, Bruno Zamborlin, and Fabrice Guédy. 2011. Online Gesture Analysis and Control of Audio Processing. In Musical Robots and Interactive Multimodal Systems. Springer, 127-142.

[7] Dominik Bial, Thorsten Appelmann, Enrico Rukzio, and Albrecht Schmidt. 2012 Improving cyclists training with tactile feedback on feet. In International Conference on Haptic and Audio Interaction Design. Springer, 41-50.

[8] Jordi Bolíbar and Roberto Bresin. 2012. Sound feedback for the optimization of performance in running. Proceedings of the Sound and Music Computing (2012) $39-41$.

[9] Robert Jan Bood, Marijn Nijssen, John Van Der Kamp, and Melvyn Roerdink 2013. The power of auditory-motor synchronization in sports: enhancing running performance by coupling cadence with the right beats. PloS one 8, 8 (2013).

[10] Jeffrey Boyd and Andrew Godbout. 2010. Corrective Sonic Feedback for Speed Skating: A Case Study. Georgia Institute of Technology.

[11] Jeffrey E Boyd, Andrew Godbout, and Chris Thornton. 2012. In situ motion capture of speed skating: Escaping the treadmill. In 2012 Ninth Conference on Computer and Robot Vision. IEEE, 460-467.

[12] Virginia Braun and Victoria Clarke. 2012. Thematic analysis. (2012)

[13] Kristin Carlson, Sarah Fdili Alaoui, Greg Corness, and Thecla Schiphorst. 2019 Shifting Spaces: Using Defamiliarization to Design Choreographic Technologies That Support Co-Creation. In Proceedings of the 6th International Conference on Movement and Computing (Tempe, AZ, USA) (MOCO '19). Association for Computing Machinery, New York, NY, USA, Article 17, 8 pages. https://doi.org/ $10.1145 / 3347122.3347140$

[14] Daniel Cesarini, Thomas Hermann, and Bodo Ungerechts. 2014. A real-time auditory biofeedback system for sports swimming. In Proceedings of the 20th International Conference on Auditory Display (ICAD 2014).

[15] Continuum. 2020. Continuum Fingerboard. https://www.hakenaudio.com/ continuum-fingerboard. Accessed 2020-08-20.

[16] Thomas D Cook, Donald Thomas Campbell, and Arles Day. 1979. Quasiexperimentation: Design \& analysis issues for field settings. Vol. 351. Houghton Mifflin Boston.

[17] Artem Dementyev, Hsin-Liu (Cindy) Kao, and Joseph A. Paradiso. 2015. SensorTape: Modular and Programmable 3D-Aware Dense Sensor Network on a Tape. In Proceedings of the 28th Annual ACM Symposium on User Interface Software \& Technology (Charlotte, NC, USA) (UIST '15). Association for Computing Machinery,
New York, NY, USA, 649-658. https://doi.org/10.1145/2807442.2807507

[18] Christopher Dobrian and Daniel Koppelman. 2006. The'E'in NIME: Musical Expression with New Computer Interfaces.. In NIME, Vol. 6. 277-282.

[19] Cirque du Soleil. 2020. Cirque du Soleil. https://www.cirquedusoleil.com. Accessed 2020-08-20.

[20] Gaël Dubus and Roberto Bresin. 2013. A systematic review of mapping strategies for the sonification of physical quantities. PloS one 8, 12 (2013), e82491.

[21] Alfred Effenberg, Ursula Fehse, and Andreas Weber. 2011. Movement Sonification: Audiovisual benefits on motor learning. In $B I O$ web of conferences, Vol. 1. EDP Sciences, 00022.

[22] Alfred O Effenberg. 2005. Movement sonification: Effects on perception and action. IEEE multimedia 12, 2 (2005), 53-59.

[23] Don Samitha Elvitigala, Denys J.C. Matthies, Löic David, Chamod Weerasinghe, and Suranga Nanayakkara. 2019. GymSoles: Improving Squats and Dead-Lifts by Visualizing the User's Center of Pressure. In Proceedings of the 2019 CHI Conference on Human Factors in Computing Systems (Glasgow, Scotland Uk) (CHI '19). Association for Computing Machinery, New York, NY, USA, 1-12. https://doi.org/10.1145/3290605.3300404

[24] Rebecca Fiebrink and Perry R Cook. 2010. The Wekinator: a system for real-time, interactive machine learning in music. In Proceedings of The Eleventh International Society for Music Information Retrieval Conference (ISMIR 2010)(Utrecht).

[25] Jérémie Garcia, Theophanis Tsandilas, Carlos Agon, and Wendy E. Mackay. 2014. Structured Observation with Polyphony: A Multifaceted Tool for Studying Music Composition. In Proceedings of the 2014 Conference on Designing Interactive Systems (Vancouver, BC, Canada) (DIS '14). Association for Computing Machinery, New York, NY, USA, 199-208. https://doi.org/10.1145/2598510.2598512

[26] Nan-Wei Gong, Steve Hodges, and Joseph A. Paradiso. 2011. Leveraging Conductive Inkjet Technology to Build a Scalable and Versatile Surface for Ubiquitous Sensing. In Proceedings of the 13th International Conference on Ubiquitous Computing (Beijing, China) (UbiComp '11). Association for Computing Machinery, New York, NY, USA, 45-54. https://doi.org/10.1145/2030112.2030120

[27] Nan-Wei Gong, Jürgen Steimle, Simon Olberding, Steve Hodges, Nicholas Edward Gillian, Yoshihiro Kawahara, and Joseph A. Paradiso. 2014. PrintSense: A Versatile Sensing Technique to Support Multimodal Flexible Surface Interaction. In Proceedings of the SIGCHI Conference on Human Factors in Computing Systems (Toronto, Ontario, Canada) (CHI '14). Association for Computing Machinery, New York, NY, USA, 1407-1410. https://doi.org/10.1145/2556288.2557173

[28] Michael Gurevich and Jeffrey Treviño. 2007. Expression and its discontents: toward an ecology of musical creation. In Proceedings of the 7th international conference on New interfaces for musical expression. 106-111.

[29] Elisabet Hagert. 2010. Proprioception of the wrist joint: a review of current concepts and possible implications on the rehabilitation of the wrist. Fournal of Hand Therapy 23, 1 (2010), 2-17.

[30] Shoichi Hasegawa, Seiichiro Ishijima, Fumihiro Kato, Hironori Mitake, and Makoto Sato. 2012. Realtime Sonification of the Center of Gravity for Skiing. In Proceedings of the 3rd Augmented Human International Conference (Megève, France) (AH '12). Association for Computing Machinery, New York, NY, USA, Article 11, 4 pages. https://doi.org/10.1145/2160125.2160136

[31] Thomas Hermann and Sebastian Zehe. 2011. Sonified aerobics-interactive sonification of coordinated body movements. International Community for Auditory Display.

[32] Oliver Höner, Thomas Hermann, and Christian Grunow. 2004. Sonification of group behavior for analysis and training of sports tactics. In Proceedings of the International Workshop on Interactive Sonification.

[33] Kristina Höök, Baptiste Caramiaux, Cumhur Erkut, Jodi Forlizzi, Nassrin Hajinejad, Michael Haller, Caroline Hummels, Katherine Isbister, Martin Jonsson, George Khut, et al. 2018. Embracing first-person perspectives in soma-based design. In Informatics, Vol. 5. Multidisciplinary Digital Publishing Institute, 8.

[34] Jessica Hummel, Thomas Hermann, Christopher Frauenberger, and Tony Stockman. 2010. Interactive sonification of german wheel sports. In Proceedings of ISon 2010-Interactive Sonification Workshop: Human Interaction with Auditory Displays.

[35] Andy Hunt, Marcelo M Wanderley, and Matthew Paradis. 2003. The importance of parameter mapping in electronic instrument design. Fournal of New Music Research 32, 4 (2003), 429-440.

[36] Atsuki Ikeda, Yuka Tanaka, Dong-Hyun Hwang, Homare Kon, and Hideki Koike. 2019. Golf Training System Using Sonification and Virtual Shadow. In ACM SIGGRAPH 2019 Emerging Technologies (Los Angeles, California) (SIGGRAPH '19). Association for Computing Machinery, New York, NY, USA, Article 14, 2 pages. https://doi.org/10.1145/3305367.3327993

[37] Shahram Izadi, Richard A. Newcombe, David Kim, Otmar Hilliges, David Molyneaux, Steve Hodges, Pushmeet Kohli, Jamie Shotton, Andrew J. Davison, and Andrew Fitzgibbon. 2011. KinectFusion: Real-Time Dynamic 3D Surface Reconstruction and Interaction. In ACM SIGGRAPH 2011 Talks (Vancouver, British Columbia, Canada) (SIGGRAPH '11). Association for Computing Machinery, New York, NY, USA, Article 23, 1 pages. https://doi.org/10.1145/2037826.2037857

[38] Raine Kajastila, Leo Holsti, and Perttu Hämäläinen. 2016. The Augmented Climbing Wall: High-Exertion Proximity Interaction on a Wall-Sized Interactive Surface. 
In Proceedings of the 2016 CHI Conference on Human Factors in Computing Systems (San Jose, California, USA) (CHI '16). Association for Computing Machinery, New York, NY, USA, 758-769. https://doi.org/10.1145/2858036.2858450

[39] Janin Koch, Nicolas Taffin, Andrés Lucero, and Wendy E. Mackay. 2020. SemanticCollage: Enriching Digital Mood Board Design with Semantic Labels. In Proceedings of the 2020 ACM Designing Interactive Systems Conference (Eindhoven, Netherlands) (DIS '20). Association for Computing Machinery, New York, NY, USA, 407-418. https://doi.org/10.1145/3357236.3395494

[40] Christin Kohrs, Nicole Angenstein, and André Brechmann. 2016. Delays in human-computer interaction and their effects on brain activity. PloS one 11, 1 (2016), e0146250.

[41] Niilo Konttinen, Kaisu Mononen, Jukka Viitasalo, and Toni Mets. 2004. The effects of augmented auditory feedback on psychomotor skill learning in precision shooting. Fournal of Sport and Exercise Psychology 26, 2 (2004), 306-316.

[42] Guillaume Lemaitre, Olivier Houix, Patrick Susini, Yon Visell, and Karmen Franinović. 2012. Feelings elicited by auditory feedback from a computationally augmented artifact: The flops. IEEE Transactions on Affective Computing 3, 3 (2012), 335-348

[43] LinnStrument. 2020. LinnStrument. https://www.rogerlinndesign.com/ linnstrument. Accessed 2020-08-20.

[44] Lian Loke and Toni Robertson. 2013. Moving and Making Strange: An Embodied Approach to Movement-Based Interaction Design. ACM Trans. Comput.-Hum. Interact. 20, 1, Article 7 (April 2013), 25 pages. https://doi.org/10.1145/2442106. 2442113

[45] Valerio Lorenzoni, Jacob Staley, Thierry Marchant, Kelsey E Onderdijk, Pieter-Jan Maes, and Marc Leman. 2019. The sonic instructor: A music-based biofeedback system for improving weightlifting technique. Plos one 14, 8 (2019), e0220915.

[46] Pieter-Jan Maes, Valerio Lorenzoni, and Joren Six. 2019. The SoundBike: musical sonification strategies to enhance cyclists' spontaneous synchronization to external music. Journal on Multimodal User Interfaces 13, 3 (2019), 155-166.

[47] Richard A Magill and David I Anderson. 2007. Motor learning and control: Concepts and applications. Vol. 11. McGraw-Hill New York.

[48] Shareen Mahmud, Jessalyn Alvina, Parmit K. Chilana, Andrea Bunt, and Joanna McGrenere. 2020. Learning Through Exploration: How Children, Adults, and Older Adults Interact with a New Feature-Rich Application. In Proceedings of the 2020 CHI Conference on Human Factors in Computing Systems (Honolulu, HI USA) (CHI '20). Association for Computing Machinery, New York, NY, USA, 1-14 https://doi.org/10.1145/3313831.3376414

[49] Claire F Michaels and Claudia Carello. 1981. Direct perception. Prentice-Hall Englewood Cliffs, NJ.

[50] E. Miranda and M. Wanderley. 2006. New Digital Musical Instruments: Control and Interaction beyond the Keyboard. A-R Editions.

[51] Florian Mueller and Katherine Isbister. 2014. Movement-Based Game Guidelines. In Proceedings of the SIGCHI Conference on Human Factors in Computing Systems (Toronto, Ontario, Canada) (CHI '14). Association for Computing Machinery, New York, NY, USA, 2191-2200. https://doi.org/10.1145/2556288.2557163

[52] Florian "Floyd" Mueller and Matthew Muirhead. 2015. Jogging with a Quadcopter. In Proceedings of the 33rd Annual ACM Conference on Human Factors in Computing Systems (Seoul, Republic of Korea) (CHI '15). Association for Computing Machinery, New York, NY, USA, 2023-2032. https://doi.org/10.1145/2702123.2702472

[53] Michael J Muller and Sarah Kuhn. 1993. Participatory design. Commun. ACM 36, 6 (1993), 24-28

[54] Guthman musical instrument competition. 2020. Guthman musical instrument competition. https://guthman.gatech.edu. Accessed 2020-08-20.

[55] Joseph W Newbold, Nadia Bianchi-Berthouze, and Nicolas E Gold. 2017. Musical expectancy in squat sonification for people who struggle with physical activity. Georgia Institute of Technology.

[56] Stina Nylander, Alex Kent, and Jakob Tholander. 2014. Swing Sound: Experiencing the Golf Swing through Sound. In CHI '14 Extended Abstracts on Human Factors in Computing Systems (Toronto, Ontario, Canada) (CHI EA '14). Association for Computing Machinery, New York, NY, USA, 443-446. https://doi.org/10.1145/ 2559206.2574789

[57] Stina Nylander, Jakob Tholander, and Alex Kent. 2013. Peripheral interaction for sports-exploring two modalities for real-time feedback. (2013).

[58] William K Ogard. 2011. Proprioception in sports medicine and athletic conditioning. Strength \& Conditioning fournal 33, 3 (2011), 111-118.

[59] OptiTrack. 2020. OptiTrack Motion Capture System. https://optitrack.com/ Accessed 2020-08-20.

[60] Joseph A Paradiso and Neil Gershenfeld. 1997. Musical applications of electric field sensing. Computer music journal 21, 2 (1997), 69-89.

[61] Hyung Kun Park and Woohun Lee. 2016. Motion Echo Snowboard: Enhancing Body Movement Perception in Sport via Visually Augmented Feedback. In Proceedings of the 2016 ACM Conference on Designing Interactive Systems (Brisbane, QLD, Australia) (DIS '16). Association for Computing Machinery, New York, NY, USA, 192-203. https://doi.org/10.1145/2901790.2901797

[62] Hyung Kun Park, HyeonBeom Yi, and Woohun Lee. 2017. Recording and Sharing Non-Visible Information on Body Movement While Skateboarding. In Proceedings of the 2017 CHI Conference on Human Factors in Computing Systems (Denver,
Colorado, USA) (CHI '17). Association for Computing Machinery, New York, NY, USA, 2488-2492. https://doi.org/10.1145/3025453.3025476

[63] Sebastiaan Pijnappel and Florian "Floyd" Mueller. 2014. Designing Interactive Technology for Skateboarding. In Proceedings of the 8th International Conference on Tangible, Embedded and Embodied Interaction (Munich, Germany) (TEI '14). Association for Computing Machinery, New York, NY, USA, 141-148. https: //doi.org/10.1145/2540930.2540950

[64] Ivan Poupyrev, Nan-Wei Gong, Shiho Fukuhara, Mustafa Emre Karagozler, Carsten Schwesig, and Karen E. Robinson. 2016. Project Jacquard: Interactive Digital Textiles at Scale. In Proceedings of the 2016 CHI Conference on $\mathrm{Hu}$ man Factors in Computing Systems (San Jose, California, USA) (CHI '16). Association for Computing Machinery, New York, NY, USA, 4216-4227. https: //doi.org/10.1145/2858036.2858176

[65] Roberto Pugliese and Tapio Takala. 2015. Sonic trampoline: the effect of audio feedback on the user experience during an exercise of jumping. IEEE MultiMedia (2015).

[66] Katerina El Raheb, Marina Stergiou, Akrivi Katifori, and Yannis Ioannidis. 2019. Dance Interactive Learning Systems: A Study on Interaction Workflow and Teaching Approaches. 52, 3, Article 50 (June 2019), 37 pages. https://doi.org/10. $1145 / 3323335$

[67] Hesam Ramezanzade, Behrouz Abdoli, Alireza Farsi, and Mohammad Ali Sanjari. 2014. The effect of sonification modelling on perception and accuracy of performing jump shot basketball. International fournal of Sports Studies 4, 11 (2014), 1388-1392.

[68] Bruce Richardson, Krispin Leydon, Mikael Fernstrom, and Joseph A Paradiso. 2004. Z-Tiles: building blocks for modular, pressure-sensing floorspaces. In CHI'04 extended abstracts on Human factors in computing systems. 1529-1532.

[69] Daniel Roetenberg, Henk Luinge, and Per Slycke. 2009. Xsens MVN: full 6DOF human motion tracking using miniature inertial sensors. Xsens Motion Technologies BV, Tech. Rep 1 (2009)

[70] Nina Schaffert, André Engel, Sebastian Schlüter, and Klaus Mattes. 2019. The sound of the underwater dolphin-kick: developing real-time audio feedback in swimming. Displays 59 (2019), 53-62.

[71] N Schaffert, R Gehret, AO Effenberg, and K Mattes. 2008. The sonified boat motion as the characteristic rhythm of several stroke rate steps. (2008).

[72] Nina Schaffert, Thenille Braun Janzen, Klaus Mattes, and Michael H Thaut. 2019. A review on the relationship between sound and movement in sports and rehabilitation. Frontiers in psychology 10 (2019), 244.

[73] Richard A Schmidt and Craig A Wrisberg. 2008. Motor learning and performance: A situation-based learning approach. Human kinetics.

[74] Gerd Schmitz, Bahram Mohammadi, Anke Hammer, Marcus Heldmann, Amir Samii, Thomas F Münte, and Alfred O Effenberg. 2013. Observation of sonified movements engages a basal ganglia frontocortical network. BMC neuroscience 14,1 (2013), 32.

[75] Norbert Schnell, Axel Röbel, Diemo Schwarz, Geoffroy Peeters, Riccardo Borghesi, et al. 2009. MuBu and Friends-Assembling Tools for Content Based Real-Time Interactive Audio Processing in Max/MSP. In ICMC.

[76] Norbert Schnell, Diemo Schwarz, Joseph Larralde, and Riccardo Borghesi. 2017. $\mathrm{PiPo}$, a plugin interface for afferent data stream processing modules. In 18th International Society for Music Information Retrieval Conference, Suzhou, China, 2017.

[77] Diemo Schwarz, Grégory Beller, Bruno Verbrugghe, and Sam Britton. 2006. Realtime corpus-based concatenative synthesis with catart. In Proc. of the 9th Int. Conference on Digital Audio Effects, Montreal, Canada.

[78] Hugo Scurto, Wanyu Liu, Benjamin Matuszewski, Frédéric Bevilacqua, Jean-Louis Frechin, Uros Petrevski, and Norbert Schnell. 2019. Entrain: Encouraging Social Interaction in Collective Music Making. In ACM SIGGRAPH 2019 Studio (Los Angeles, California) (SIGGRAPH '19). Association for Computing Machinery, New York, NY, USA, Article 5, 2 pages. https://doi.org/10.1145/3306306.3328004

[79] Sensel. 2020. The Sensel Morph. https://sensel.com/pages/the-sensel-morph. Accessed 2020-08-20.

[80] Roland Sigrist, Samantha Fox, Robert Riener, and Peter Wolf. 2016. Benefits of crank moment sonification in cycling. Procedia Eng 147 (2016), 513-518.

[81] Roland Sigrist, Georg Rauter, Robert Riener, and Peter Wolf. 2013. Augmented visual, auditory, haptic, and multimodal feedback in motor learning: a review. Psychonomic bulletin \& review 20, 1 (2013), 21-53.

[82] Daniel Spelmezan. 2012. An Investigation into the Use of Tactile Instructions in Snowboarding. In Proceedings of the 14th International Conference on HumanComputer Interaction with Mobile Devices and Services (San Francisco, California, USA) (MobileHCI '12). Association for Computing Machinery, New York, NY, USA, 417-426. https://doi.org/10.1145/2371574.2371639

[83] Ana Tajadura-Jimenez, Nadia Bianchi-Berthouze, Enrico Furfaro, and Frederic Bevilacqua. 2015. Sonification of Surface Tapping Changes Behavior, Surface Perception, and Emotion. MultiMedia, IEEE 22, 1 (2015), 48-57. https://doi.org/ 10.1109/MMUL.2015.14

[84] Laia Turmo Vidal, Elena Márquez Segura, Christopher Boyer, and Annika Waern. 2019. Enlightened Yoga: Designing an Augmented Class with Wearable Lights to Support Instruction. In Proceedings of the 2019 on Designing Interactive Systems 
Conference (San Diego, CA, USA) (DIS '19). Association for Computing Machinery, New York, NY, USA, 1017-1031. https://doi.org/10.1145/3322276.3322338

[85] Laia Turmo Vidal, Hui Zhu, and Abraham Riego-Delgado. 2020. BodyLights: Open-Ended Augmented Feedback to Support Training Towards a Correct Exercise Execution. In Proceedings of the 2020 CHI Conference on Human Factors in Computing Systems (Honolulu, HI, USA) (CHI '20). Association for Computing Machinery, New York, NY, USA, 1-14. https://doi.org/10.1145/3313831.3376268

[86] Martin Weigel, Tong Lu, Gilles Bailly, Antti Oulasvirta, Carmel Majidi, and Jürgen Steimle. 2015. ISkin: Flexible, Stretchable and Visually Customizable On-Body Touch Sensors for Mobile Computing. In Proceedings of the 33rd Annual ACM Conference on Human Factors in Computing Systems (Seoul, Republic of Korea) (CHI '15). Association for Computing Machinery, New York, NY, USA, 2991-3000. https://doi.org/10.1145/2702123.2702391

[87] Matthew Wright, Ryan J Cassidy, and Michael Zbyszynski. 2004. Audio and Gesture Latency Measurements on Linux and OSX. In ICMC.

[88] George M Wyburn. 1964. Human senses and perception. Vol. 82. University of Toronto Press.

[89] Jiajun Yang and Andy Hunt. 2015. Real-time sonification of biceps curl exercise using muscular activity and kinematics. Georgia Institute of Technology.

[90] Thomas G. Zimmerman, Joshua R. Smith, Joseph A. Paradiso, David Allport, and Neil Gershenfeld. 1995. Applying Electric Field Sensing to Human-Computer Interfaces. In Proceedings of the SIGCHI Conference on Human Factors in Computing Systems (Denver, Colorado, USA) (CHI '95). ACM Press/Addison-Wesley Publishing Co., USA, 280-287. https://doi.org/10.1145/223904.223940 